\title{
Separating Haptic Guidance from Task Dynamics: A Practical Solution via Cutaneous Devices
}

\author{
Evan Pezent ${ }^{\dagger}$, Simone Fani ${ }^{\ddagger \S}$, Joshua Bradley ${ }^{\dagger}$, Matteo Bianchi ${ }^{\ddagger \Lambda}$, and Marcia K. O’Malley ${ }^{\dagger}$
}

\begin{abstract}
There is much interest in using haptic feedback for training new skills or guiding human movement. However, the results of studies that have incorporated haptic guidance to train new skills are mixed, depending on task complexity and the method by which the haptic guidance is implemented. Subjects show dependency on the guidance forces and difficulty in discerning which aspects of the haptic feedback are related to the task dynamics and which are meant to convey task completion strategies. For these reasons, new methods to separate haptic cues for guidance from haptic feedback of task dynamics are needed. In this experiment, 30 subjects completed a trajectory following task using a wrist exoskeleton which also rendered task forces. To assist subjects, guidance cues were provided in one of three forms: (1) cutaneous forces from a wearable skin-stretch device on the ipsilateral forearm and (2) contralateral forearm, and (3) kinesthetic forces from a kinematically similar wrist exoskeleton operated by the contralateral arm. The efficacies of each guidance condition are compared by examining subject performance and learning rates. The results indicate that cutaneous guidance is nearly as effective as kinesthetic guidance, making it a practical and cost-effective alternative for spatially separated assistance.
\end{abstract}

\section{INTRODUCTION}

Haptic devices have long been recognized for their potential and value in virtual or augmented reality training applications. The premise is that robotic or haptic devices can provide guidance to a novice subject regarding the methods or strategies that should be used to complete a task. Robotmediated training offers advantages over traditional humanmediated training, including the potential for one human expert to train a large number of novices simultaneously. In addition, the programmable nature of the training interface can allow for online modifications to keep the novice engaged and the training protocol effective and tailored to the user's performance level. There is strong evidence to support the addition of haptic cues for performance enhancement [1], [2], [3], [4], especially in dynamic tasks [5]. It has been demonstrated, however, that the combination of task forces and guidance forces relayed to the trainee simultaneously via a kinesthetic type haptic device can lead to dependence on the guidance, misinterpretation of the provided feedback, and an inability to demonstrate skill transfer [6].

This research has received funding from the National Science Foundation IGERT fellowship (NSF DGE-1250104), and from the European Union's Horizon 2020 Research and Innovation Programme under Grant Agreement No.688857 (SoftPro). ${ }^{\dagger}$ Mechatronics and Haptic Interfaces Laboratory, Dept. of Mechanical Engineering, Rice University, Houston, TX 77005, USA. ${ }^{\ddagger}$ Research Center Enrico Piaggio, University of Pisa, Largo Lucio Lazzarino 1, 56122 Pisa, Italy. ${ }^{\S}$ Dept. of Advanced Robotics, Soft Robotics for Human Cooperation and Rehabilitation research line, Istituto Italiano di Tecnologia, via Morego, 30, 16163 Genova, Italy. ${ }^{\Lambda}$ Dept. of Information Engineering, University of Pisa, via G. Caruso, 16, 56122 Pisa, Italy.

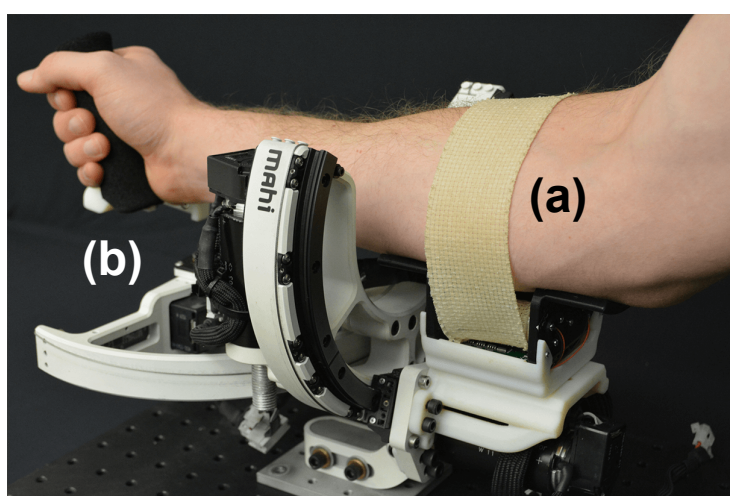

Fig. 1. Separating task and guidance forces during haptic training remains a fundamental issue in the field. This paper presents a novel approach whereby (a) a wearable skin stretch device provides cutaneous guidance forces, while haptic training is administered through (b) a powered wrist exoskeleton.

One strategy that attempts to minimize trainee misinterpretation of task and guidance uses multiple devices or points of contact with the trainee to spatially separate the two types of feedback. This approach has been categorized as "spatially separated assistance" (SSA) [6]. The first, and perhaps best example of SSA, is the double-contact paradigm proposed by Gillespie et al. [7]. It makes use of a specialized haptic device in order to present guidance from a virtual expert via one haptic channel (the back of the novice's hand) and forces arising from the task dynamics via a second channel (the novice's palm). Wulf et al. [8] showed that SSA was superior to practice without guidance in a simulated skiing task, where guidance and task forces were provided through the poles and skis, respectively. Powell and O'Malley [6] implemented SSA using two haptic joysticks. Participants controlled a virtual mass-spring system using the primary joystick, which rendered task forces, while guidance forces were displayed on the secondary joystick. This bimanual approach to training is supported by research on human motor learning, including studies that have shown that a transference of skills between bimanual and unimanual tasks can take place, even if partially [9]. Importantly, Tcheang et al. [10] showed that applying forces to one arm will not impede learning of force fields by the other arm.

Each of these examples of SSA relies on kinesthetic type feedback that requires complex and potentially expensive custom haptic devices unique to a particular task (for example, multi degree-of-freedom devices to simulate rowing [11], [12] or tennis swings [13]). Further, some types of kinesthetic haptic assistance, although performance enhancing, have been ineffective when it comes to demonstrating retention of skill or transfer to a similar task [6], [14], [15]. 
In contrast, cutaneous feedback, typically conveyed with low-cost wearable devices, has the potential to be widely applied to the training of complex movements, can be generalized to different tasks, and integrates with the human body in a very ecological manner [16], [17]. Tactile cueing systems have been extensively studied to determine appropriate methods for guiding wrist rotation movements [18], and motion guidance has been effectively conveyed through both vibrational [19] and skin stretch [20] tactile cues. Skin stretch, specifically, is thought to deliver directional information through a modality matching approach [21] similar to how a human trainer might guide a trainee's movement.

In this paper, we propose a new approach to achieving SSA that leverages multiple forms of haptic feedback. Task forces are relayed via a traditional kinesthetic haptic interface, while guidance forces are conveyed through a tactile skin stretch modality (Fig. 1). Additionally, we present an experiment that directly compares the effectiveness of SSA achieved via both cutaneous and kinesthetic guidance. We further explore the role of placing cutaneous guidance ipsilateral versus contralateral, as is necessary when using a kinematically similar kinesthetic guidance device. An overview of the devices used, the task, and the guidance conditions is provided in Section II. Results and a discussion of subject learning rates and performance under each guidance condition follows in Section III, with our conclusions summarized in Section IV.

\section{Methods}

We designed a trajectory following task displaying pertinent and non-negligible dynamic forces. Subjects used their right arm to complete the task through positional input of a wrist-forearm exoskeletal device (the Task Device), which also served to render the task forces. Two additional devices were used to provide guidance across three conditions. In the first, a kinematically similar exoskeleton (the Kinesthetic Guidance Device) provided proprioceptive guidance on the contralateral arm, while in the second and third, a skin-stretch device (the Cutaneous Guidance Device) provided guidance on either the ipsilateral or contralateral arm, respectively.

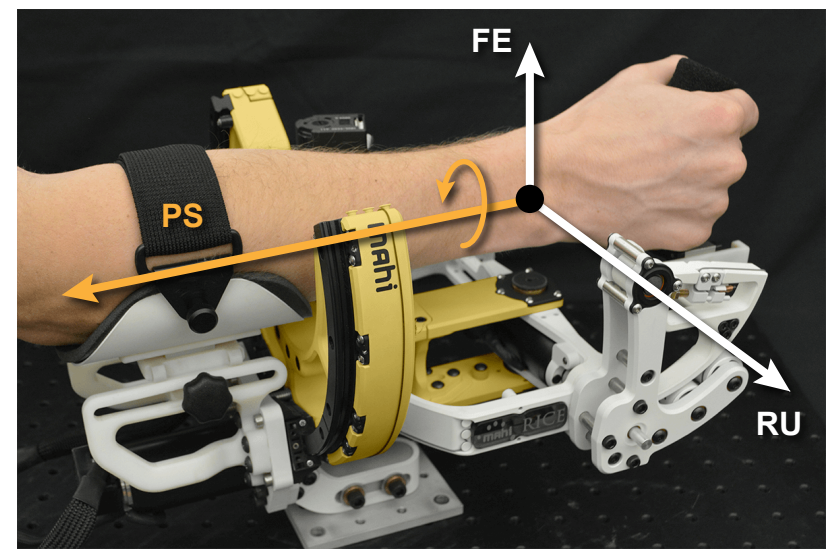

Fig. 2. The OpenWrist [22] served as the Task Device. The forearm pronation/supination (PS) DOF (yellow) was used for task input and rendering task forces, while the wrist flexion/extension (FE) and radial/ulnar (RU) deviation DOFs were locked in their neutral positions.

\section{A. Devices Used}

1) Task Device: The OpenWrist [22] was chosen as the task completion device (Fig. 2). It is a 3 degree-offreedom (DOF), purely rotational, exoskeleton designed for wrist rehabilitation, capable of rendering high fidelity haptic environments through the application of DC motors and backlash-free capstan-cable transmissions. The first joint of the exoskeleton, forearm pronation/supination (PS), was used as the driving input of the task, while the two distal joints, wrist flexion/extension (FE) and radial/ulnar deviation (RU), were locked in their neutral positions through control. Gravity and Coulomb friction compensation torques were added to the task torques so that ideally only the dynamics arising from the virtual task would be felt.

2) Kinesthetic Guidance Device: The MAHI Exo-II (ME-II) [23] was used for the kinesthetic guidance condition (Fig. 3). Similar to the OpenWrist, the ME-II is an upperextremity exoskeleton employing DC motors and capstancable transmissions. In addition to the same wrist and forearm DOFs as the OpenWrist (albeit in the form of a parallel mechanism), it features an additional DOF for the elbow. The FE and RU wrist DOFs were mechanically fixed in their neutral position and the elbow DOF was locked through control. Proprioceptive guidance was provided through the rotation of the forearm PS joint, which is similar enough to the OpenWrist's PS joint that any mechanical differences between the devices were considered irrelevant.

3) Cutaneous Guidance Device: For cutaneous guidance, we used the Clenching Upper-limb Force Feedback device (CUFF), a wearable haptic device $(123.5 \times 76 \times 80 \mathrm{~mm}, 226 \mathrm{~g})$ that distributes mechano-tactile forces on the user's skin [24] and has been successfully employed in prosthetic and augmented human-robot applications [25], [26] (Fig. 4). The device consists of a composite silicone-fabric band which is wrapped around the user's limb. Two DC motors, attached to opposite ends of the band, are independently driven in either opposite or same directions to display normal or tangential cutaneous force, respectively. For consistency, the band is pretensioned to exert a normal force of $3 \mathrm{~N}$ against the arm.

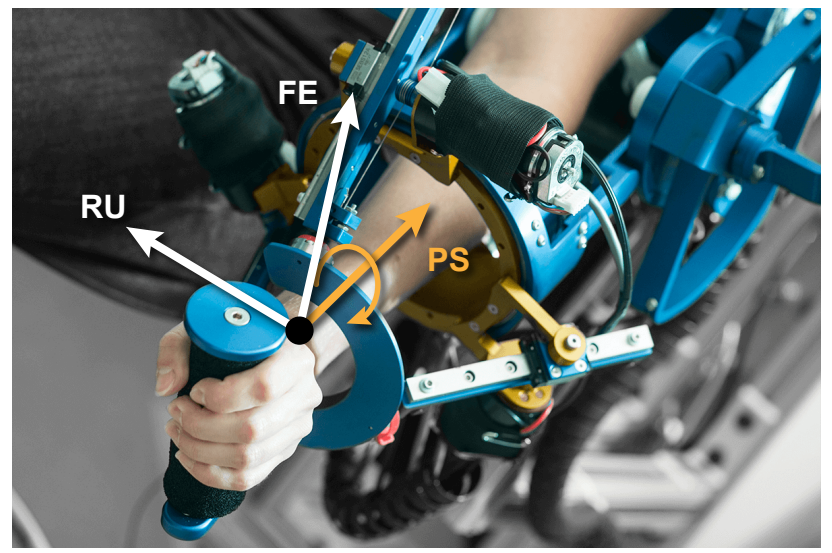

Fig. 3. The MAHI Exo-II [23] was the Kinesthetic Guidance Device The elbow DOF and parallel mechanism providing flexion/extension (FE) and radial/ulnar deviation (RU) were locked in a neutral position, while the forearm pronation/supination (PS) DOF (yellow) provided guidance. 

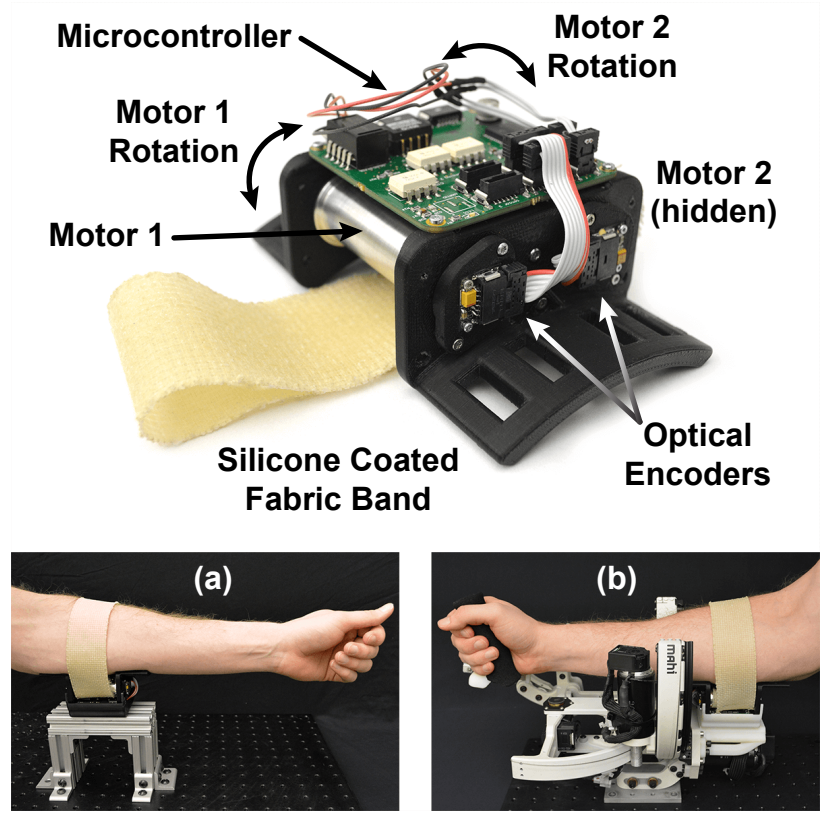

Fig. 4. The Clenching Upper-limb Force Feedback device (CUFF) [24] served as the Cutaneous Guidance Device. (a) The CUFF in the contralateral condition, mounted to an $80 / 20$ aluminum frame. Subjects were provided a hand rest to prevent fatigue (not pictured). (b) The CUFF in the ipsilateral condition, integrated directly into the OpenWrist Task Device.

While the CUFF is typically worn as an ungrounded device, it was fixed to the work surface in this experiment. In the ipsilateral condition, it was directly integrated with the OpenWrist Task Device, replacing the default forearm rest (Fig. 4-b), and in the contralateral condition, it was mounted to a frame of the same height (Fig. 4-a). A pillow hand rest was provided to prevent fatigue in the contralateral condition.

\section{B. Description of Task}

Consideration should be taken when designing a controlled task that combines haptic guidance and task dynamics with visual information. Forces should be physically relevant to the displayed visuals, and the task should necessitate haptic guidance, proving difficult or impossible without it. Furthermore, the task must be intuitive for first-time users, yet provide enough challenge so that learning occurs.

In this experiment, subjects were tasked with controlling the position of a virtual double pendulum (Fig. 5) displayed on a computer screen. The pendulum, with its first joint coupled to the OpenWrist forearm PS joint via a stiff virtual spring-damper, was simulated by numerically integrating

$$
\begin{gathered}
\tau_{1}=K\left(\theta_{\text {ow }}-\theta\right)+B\left(\dot{\theta}_{\text {ow }}-\dot{\theta}\right) \\
=\left[l_{1}^{2} m_{1}+\left(l_{1}^{2}+l_{2}^{2}+2 l_{1} l_{2} \mathrm{c}_{2}\right) m_{2}\right] \ddot{\theta}_{1}+b_{1} \dot{\theta}_{1} \\
+\left(l_{2}^{2}+l_{1} l_{2} \mathrm{c}_{2}\right) m_{2} \ddot{\theta}_{2}-l_{1} l_{2} \mathrm{~s}_{2} m_{2} \dot{\theta}_{2}^{2}-2 l_{1} l_{2} \mathrm{~s}_{2} m_{2} \dot{\theta}_{1} \dot{\theta}_{2} \\
+\left[l_{1} \mathrm{c}_{1} m_{1}+\left(l_{1} \mathrm{c}_{1}+l_{2} \mathrm{c}_{1+2}\right) m_{2}\right] g \\
\tau_{2}=\left(l_{1} l_{2} \mathrm{c}_{2}+l_{2}^{2}\right) m_{2} \ddot{\theta}_{1}+l_{1} l_{2} \mathrm{~s}_{2} m_{2} \dot{\theta}_{1}^{2} \\
+l_{2}^{2} m_{2} \ddot{\theta}_{2}+b_{2} \dot{\theta}_{2}+l_{2} \mathrm{c}_{1+2} m_{2} g=0
\end{gathered}
$$

where $\theta_{1}, \theta_{2}$, and their derivatives are the pendulum state, $\mathrm{s}_{i}$ is $\sin \theta_{i}, \mathrm{c}_{i}$ is $\cos \theta_{i}, \tau_{1}$ and $\tau_{2}$ are the pendulum joint torques, and $\theta_{o w}$ and $\dot{\theta}_{o w}$ are the position and velocity of the
OpenWrist forearm PS joint. The coupling stiffness $K$ and damping $B$ were $15 \mathrm{Nm} / \mathrm{rad}$ and $1 \mathrm{Nm}$-s/rad, respectively. While maintaining control of the first link, subjects could feel the pendulum reaction torque $\tau_{1}$, which was rendered with the OpenWrist. The pendulum parameters $\left(m_{1}=10 \mathrm{~g}\right.$, $m_{2}=150 \mathrm{~g}, l_{1}=45 \mathrm{~cm}, l_{2}=30 \mathrm{~cm}$, and $b_{1}=b_{2}=1 \mathrm{mNm}$ - $\left./ / \mathrm{rad}\right)$ were chosen during pilot trials so that the pendulum was relatively dynamic, but not fatiguing for subjects to handle. In this way, our task design provided meaningful forces that were easily understood by the user, while remaining non-negligible.

While in control of the double pendulum, users were asked to follow a continuous angular trajectory $\theta_{r e f}$ with the first link of the pendulum. The equation for the reference trajectory was computed through the summation of sine waves (Eqn. 3) and further normalized (Eqn. 4) to an amplitude of \pm 30 degrees, the largest forearm rotation that would not result in subject fatigue. Three unique sets of equation parameters (Table I) were chosen to represent Easy, Medium, and Hard trajectories (Fig. 6). Parameters were heuristically selected and tested during pilot trials under the intuition that increased frequency and randomness would elevate difficulty.

$$
\begin{gathered}
\theta_{r e f}^{\prime}(t)=A \sin (2 \pi a t)+B \sin (2 \pi b t)+C \sin (2 \pi c t) \\
\theta_{r e f}(t)=30^{\circ} \frac{\theta_{r e f}^{\prime}(t)}{\max \theta_{r e f}^{\prime}}
\end{gathered}
$$

The trajectory itself was not directly displayed to the subject, since this would have trivialized the task. Instead, a visual indicator in the form of a light on the second pendulum joint indicated proximity to the trajectory reference angle. The brightness of the light increased as the subject approached the reference angle, or decreased their angular error. Thus, in the visual absence of the reference trajectory, the subject's objective was to keep the light shining as brightly as possible throughout each trial.

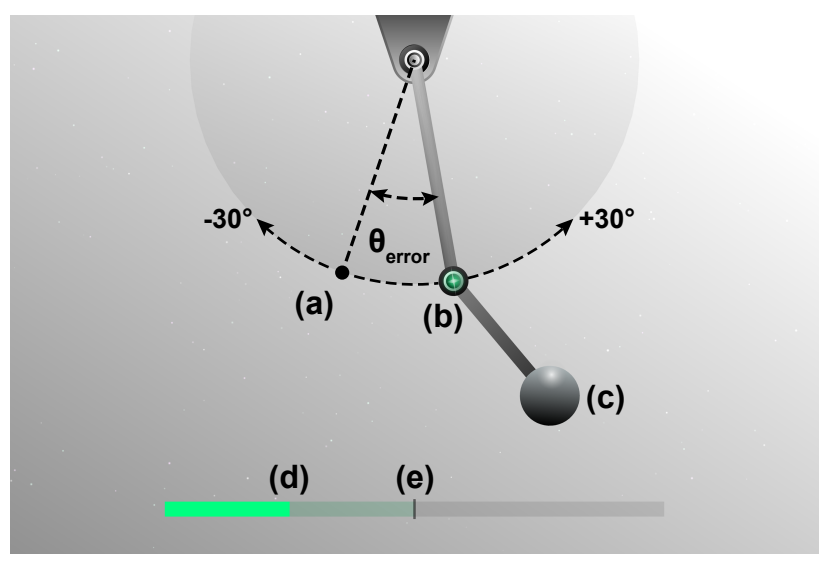

Fig. 5. Task Visualization - The angular reference trajectory, represented by the black expert dot (a) moves along the dashed arc. Note that the expert dot is shown to the user during a familiarization period, but hidden from view in all subsequent trials. The subject rotates the first pendulum link, moving the location of the indicator light (b). The indicator light intensity increases as $\boldsymbol{\theta}_{\text {error }}$ shrinks. Thus, the objective was to keep the light shining as brightly as possible at all times. The second, freely rotating pendulum link and attached mass (c) contribute to dynamic task forces. The score bar (d) fills as the user maintains close proximity to trajectory reference angle, and a tic mark (e) shows the user's previous high score for the current trajectory. 
TABLE I

TRAJECTORY PARAMETERS

\begin{tabular}{lcccccc} 
Difficulty & A & B & C & a $[\mathbf{H z}]$ & b $[\mathbf{H z}]$ & $\mathbf{c}[\mathbf{H z}]$ \\
\hline Easy & 1.0 & 0.0 & 0.0 & 0.20 & 0.0 & 0.0 \\
Medium & -1.0 & -0.5 & 0.0 & 0.25 & 0.1 & 0.0 \\
Hard & 0.5 & -0.5 & -1.0 & 0.20 & 0.1 & 0.4
\end{tabular}

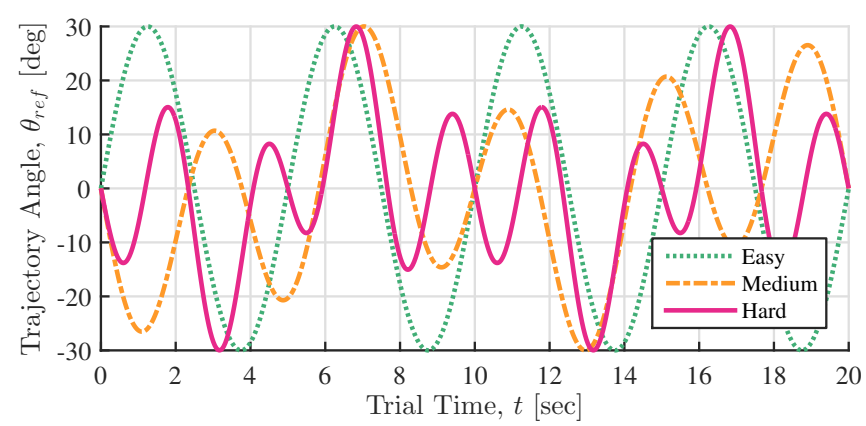

Fig. 6. The angular trajectories subjects were required to follow.

To keep subjects engaged, a score bar was shown at the bottom of the screen. The scoring formula, given by

$$
\text { score }=\sum_{t=0}^{T} \max \left(0, \min \left(10,10-\left|\theta_{\text {error }}(t)\right|\right)\right)
$$

where $\theta_{\text {error }}(t)=\theta_{\text {ow }}(t)-\theta_{\text {ref }}(t)$ is calculated in degrees, rewarded players for remaining below $10^{\circ}$ of error, a challenging but feasible feat. The score bar was reset with each new trial, and the subject's high score for the current trajectory was shown to encourage continuous improvement.

\section{Guidance Conditions}

The visual feedback from the pendulum indicator light alone did not facilitate successful completion of the task. While it provided proximal feedback, it did not convey which direction to move, thus necessitating haptic guidance. Three separate haptic guidance conditions were tested:

CI - Cutaneous Ipsilateral guidance from the CUFF

CC - Cutaneous Contralateral guidance from the CUFF

KC - Kinesthetic Contralateral guidance from the ME-II Note that a curtain occluded the subject's view of each guidance device to prevent unintended use of visual information.

In an effort to make guidance intuitive, a feed-forward paradigm was selected in which the magnitude of guidance cues was proportional to the trajectory reference angle. The decision to provide feed-forward instead of feedback guidance was made so that interpreting visual feedback from the pendulum light would remain necessary. In the case of CUFF-based guidance, both motors rotated in the same direction to generate tangential forces on the subject's forearm. Note that in condition CI the CUFF forces were not enough to cause rotation of the forearm or Task Device, nor did voluntary forearm rotation significantly affect the consistency of CUFF forearm skin stretch. The motor position gain (250 ticks/deg) was chosen in pilot trials so that the band rotation would approximately match the angular trajectory. Similarly, the forearm DOF of the ME-II followed the trajectory reference angle through position control.
It is important to discuss the choice of position-controlled guidance. With the CUFF, motor positions are commanded to move the belt which, due to contact friction and skin elasticity, imparts a shear force. Consequently, we decided that the ME-II should also employ position control, rather than torque control, in order to maintain consistency across guidance conditions. Furthermore, torque guidance would be counterintuitive since the ME-II's forearm joint angle would inadvertently drift over time, causing confusion for any subjects who might be relying on proprioceptive information. To mimic the elastic nature of the CUFF-skin interaction, the PD position controller on the ME-II was tuned to display a low impedance $\left(K_{P}=4 \mathrm{Nm} / \mathrm{rad}, K_{D}=0.05 \mathrm{Nm}\right.$-s/rad), which also minimized subject discomfort.

\section{Experimental Design and Subjects}

The study was performed during one-hour sessions which consisted of a two minute familiarization period and 72, 20 second long trials. Each subject received only one of the three guidance conditions. During the familiarization phase, subjects were shown the reference trajectory as a visible expert dot moving on the screen (as in Fig. 5-a), and were instructed to associate the feeling of the haptic guidance with the expert dot location and pendulum indicator light brightness. They were informed that in future trials, the expert dot would be hidden, and they would be forced to find the path based solely on the haptic guidance and the indicator light. After familiarization, subjects completed six blocks of trials. Each block consisted of four Easy, four Medium, and four Hard trials shuffled randomly. Depending on which trajectory was presented, the pendulum indicator light and score bar would change color to green (Easy), yellow (Medium), or red (Hard) so that subjects could learn the different trajectories as separate entities. After 36 trials, subjects were given the option to take a five minute break and then returned for another three blocks of training.

A total of 30 subjects enrolled in the experiment and were divided evenly among the three experimental conditions. All subjects, (22 male, 8 female, ages 19 to 21 ) were righthanded with no significant visual deficiencies (specifically, colorblindness) or motor impairments. Each subject provided informed consent according to the policies of the the Rice University Institutional Review Board (IRB-FY-2018-29).

\section{E. Data Analysis}

We used a uni-variate type III repeated measures ANOVA to investigate trends in subject mean error in the results from our mixed-design experiment. Specifically, we wanted to determine if a significant interaction involving condition existed, which would indicate if any guidance method was more effective than others. Running the KolmogorovSmirnov normality test on each set of block means separately revealed that the data were normally distributed, with all $p>$.07. Since the data were non-spherical, the HuynhFeldt epsilon was calculated to correct the $p$ values for the ANOVA. In order to investigate more specific trends in the data, $t$-tests were used assuming (and correcting for) heterogeneous variance between groups with Welch's adjustment. 
Since multiple statistical tests were performed, the False Detection Rate (FDR) correction was applied to the critical alpha level of $\alpha=.05$ to reduce the family-wise type I error rate. This correction recovers some of the statistical power that is lost unnecessarily due to the Bonferroni correction [27]. For a given number of statistical tests, $c$, the FDR correction is given by the following formula: $p_{k} \leq \frac{k}{c} \alpha$, where the $p$-values are sorted from smallest to largest, and the $k$ th $p$-value is compared to the $k$ th FDR corrected $\alpha$ level.

\section{RESUlTS AND DISCUSSION}

The main results are shown in Fig. 7, where absolute trajectory error, grouped by block number, is averaged across all subjects. It appears that a learning curve across blocks emerges for all conditions before saturating near block 4 . This is supported by the ANOVA, which revealed that the main effect of block was significant $(F(2.8,76.0)=$ $39.88, p<.001)$. Because the interaction of condition and block was found to be reliable in the omnibus ANOVA, block averages of absolute trajectory error were collapsed across levels of difficulty (Fig. III-a). We calculated these values to identify how the effect of block (i.e. learning curve) is different between all conditions. Pair-wise $t$-tests were conducted on linear contrasts created from the block averages. This allowed us to determine whether the slopes of the learning curves were different between conditions.

Comparing combined ipsilateral and contralateral cutaneous guidance (purple dash-dot line) with kinesthetic guidance (blue solid line), we can see that cutaneous guidance required more learning since error under these conditions was initially higher and drops with a steeper slope than the kinesthetic guidance condition $(t(9)=2.91, p=.02)$. Performance under kinesthetic guidance improved very little over the course of the experiment. This was expected, as most individuals are more accustomed to interpreting proprioceptive information than cutaneous information. An interesting result is that performance under cutaneous guidance appears to converge towards that of kinesthetic guidance. In fact, no statistically significant difference could be detected between the mean error in the combined cutaneous conditions and the kinesthetic guidance condition for blocks 4-6, with $p=.25$. Considering the cutaneous guidance conditions separately, it is still possible to detect a difference in slopes between cutaneous and kinesthetic guidance $(t(15.1)=3.17, p=$ .006 for $\mathrm{CI}$ and $\mathrm{KC}$, and $t(11.2)=2.72, p=.02$ for $\mathrm{CC}$ and $\mathrm{KC}$ ). It is reasonable to conclude that, given enough time and training, these trends would continue and that individuals may be able to use cutaneous guidance nearly as effectively as kinesthetic guidance. This is an exciting result for situations in which separated guidance is needed, but the costs or ease of providing kinesthetic guidance with a kinematically similar device is prohibitive. The CUFF and similar cutaneous devices can provide inexpensive and practical solutions to separating guidance that is easily integrated with the training protocol and adaptable to real world applications.

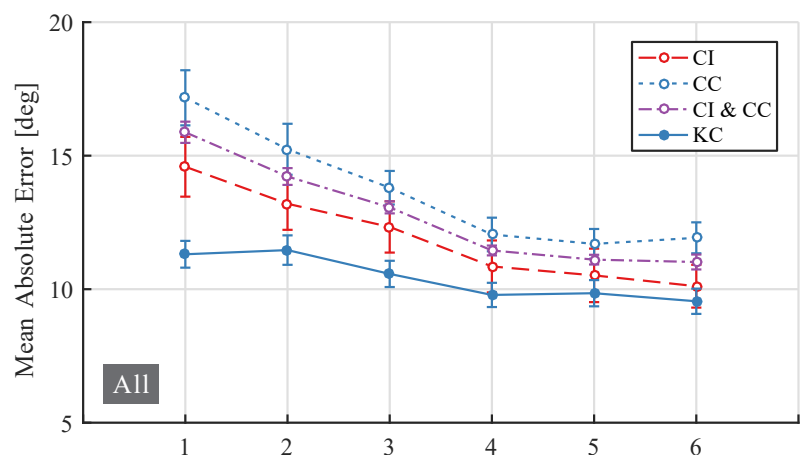

(a)

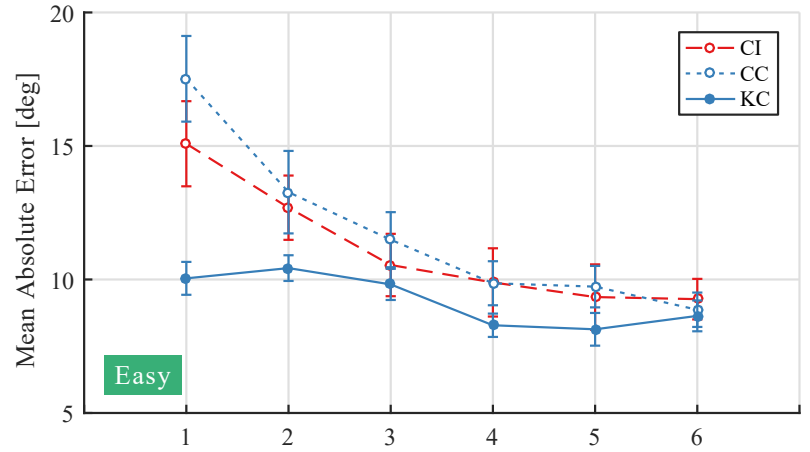

(b)

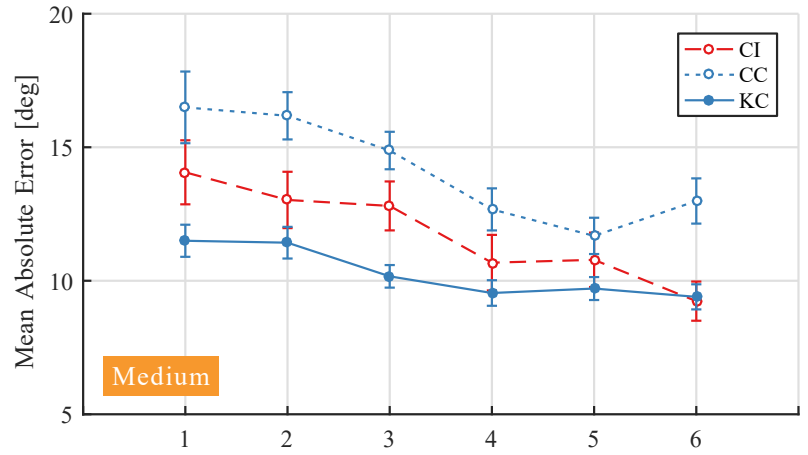

(c)

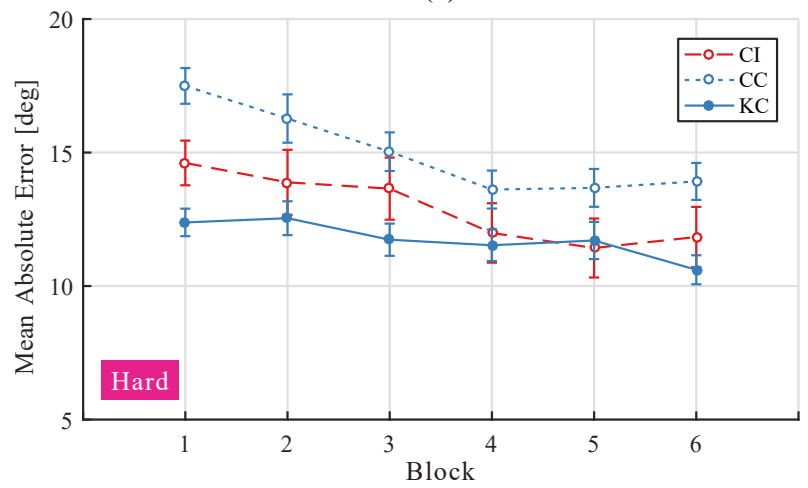

(d)

Fig. 7. Results - The mean trajectory error (with error bars denoting the 95\% confidence interval) is depicted. (a) Averaging across block and difficulty, error plateaus at approximately 12 degrees. Although all conditions improve with training, the slope of the learning curves between conditions are significantly different. (b) The plateau for error is lowest for Easy trajectories $(t(29)=4.42, p<.001)$, and the slope is statistically steeper than the slopes of the other learning curves $(t(29)=2.38, p=.024)$. (c) The learning curves for the Medium trajectory plateau higher than Easy but lower than Hard. (d) Performance on the Hard trajectories plateaus quickly. 
Separating cutaneous guidance into its ipsilateral and contralateral conditions seems to reveal that subjects performed better with ipsilateral guidance. This general observation compliments the work of Brown et. al [28], which concluded that co-locating feedback and action was more effective than non co-locating them. However, the difference represents only a trend, as the slopes and final values of conditions $\mathrm{CI}$ and $\mathrm{CC}$ were not statistically different.

Subjects plateau at lower levels of performance when the task difficulty increases. In the Easy trials (Fig. 7-b), the mean error plateaus below 10 degrees, while in the Medium (Fig. 7-c) and Hard (Fig. 7-d) trials, the mean error stays between 10 and 15 degrees in the final blocks. However, these differences are not statistically significant. The largest pairwise difference was between the Easy and Hard difficulty plateaus $(t(9)=1.8, p=.10)$. The main effect of difficulty was significant $(F(1.3,34.0)=12.31, p<.001)$, as was the interaction of block and difficulty $(F(6.4,172.0)=$ $2.34, p=.031)$. The significance of block and difficulty as main effects implies that learning occurred, and that the average performance is different for each level of path difficulty. The interaction of block and difficulty implies that there is a significant difference in learning rate between at least two difficulty levels. However, the performance plateaus at a similar level for every difficulty, and this indicates that the CUFF is a viable guidance device compared to the exoskeleton, even for difficult tasks.

\section{CONCLUSION}

This paper presents a thorough comparison of three SSA schemes for haptic training. More specifically, task forces and guidance forces were separated, with task forces provided through an exoskeleton, and guidance forces provided either through a kinematically similar exoskeleton on the contralateral arm or a wearable skin stretch device on the ipsilateral or contralateral arm. While each condition promoted increased scoring rates as time progressed, comparisons of subject performance measured through either accumulated error or score revealed a signficant effect of guidance condition. Given enough time and training, individuals may be able to learn and use cutaneous guidance nearly as effectively as kinesthetic guidance, offering a more ecological integration of haptic guidance with the user's body and a more economic and generalized approach than those offered by prohibitive kinesthetic devices. Furthermore, ipsilateral and contralateral cutaneous guidance conditions failed to show any significant difference. Overall, our results are very promising since they suggest a novel way of providing haptic feedback in SSA through wearable cutaneous devices.

\section{REFERENCES}

[1] S. A. Bowyer, B. L. Davies, and F. R. y Baena, "Active constraints/virtual fixtures: A survey," IEEE Trans. on Robot., vol. 30, no. 1, pp. 138-157, Feb 2014.

[2] K. S. Hale and K. M. Stanney, "Deriving haptic design guidelines from human physiological, psychophysical, and neurological foundations," IEEE Comput. Graphics and Appl., vol. 24, no. 2, pp. 33-39, 2004.

[3] L. Marchal-Crespo and D. J. Reinkensmeyer, "Review of control strategies for robotic movement training after neurologic injury," $J$. of Neuroeng. and Rehabil., vol. 6, no. 1, p. 20, 2009.
[4] R. Sigrist et al., "Augmented visual, auditory, haptic, and multimodal feedback in motor learning: A review," Psychonomic Bulletin \& Review, vol. 20, no. 1, pp. 21-53, 2013.

[5] M. K. O'Malley et al., "Shared control in haptic systems for performance enhancement and training," J. Dynamic Systems, Measurement, and Control, vol. 128, no. 1, pp. 75-85, 2006.

[6] D. Powell and M. K. O'Malley, "The task-dependent efficacy of shared-control haptic guidance paradigms," IEEE Trans. Haptics, vol. 5, no. 3, pp. 208-219, Third 2012.

[7] R. B. Gillespie et al., "The virtual teacher," in Proceedings of the ASME Dynamic Systems and Control Division, vol. 64, 1998, pp. 171178.

[8] G. Wulf, C. Shea, and C. A. Whitacre, "Physical-guidance benefits in learning a complex motor skill," J. of Motor Behavior, vol. 30, pp. 367-80, 121998.

[9] D. Nozaki et al., "Limited transfer of learning between unimanual and bimanual skills within the same limb," Nature Neuroscience, vol. 9, no. 11, pp. 1364-1366, 2006.

[10] L. Tcheang et al., "Simultaneous bimanual dynamics are learned without interference," Experimental Brain Research, vol. 183, no. 1, pp. 17-25, 2007.

[11] G. Rauter et al., "A tendon-based parallel robot applied to motor learning in sports," in Intl. Conf. Biomed. Robot. and Biomechatronics (BioRob), 2010, pp. 82-87.

[12] J. von Zitzewitz et al., "Real-time rowing simulator with multimodal feedback," Sports Technology, vol. 1, no. 6, pp. 257-266, 2008.

[13] L. Marchal-Crespo et al., "The effect of haptic guidance and visual feedback on learning a complex tennis task," Experimental Brain Research, vol. 231, no. 3, pp. 277-291, 2013.

[14] Y. Li et al., "Negative efficacy of fixed gain error reducing shared control for training in virtual environments," ACM Trans. on Applied Perception (TAP), vol. 6, no. 1, p. 3, 2009.

[15] L. M. Crespo and D. J. Reinkensmeyer, "Haptic guidance can enhance motor learning of a steering task," J. Motor Behavior, vol. 40, no. 6, pp. $545-557,2008$.

[16] M. Bianchi, "A fabric-based approach for wearable haptics," Electronics, vol. 5, no. 3, p. 44, 2016.

[17] C. Pacchierotti et al., "Wearable haptic systems for the fingertip and the hand: Taxonomy, review, and perspectives," IEEE Trans. Haptics, 2017.

[18] A. A. Stanley and K. J. Kuchenbecker, "Evaluation of tactile feedback methods for wrist rotation guidance," IEEE Trans. Haptics, vol. 5, no. 3, pp. 240-251, 2012.

[19] K. Bark et al., "Effects of vibrotactile feedback on human learning of arm motions," IEEE Trans. Neural Syst. and Rehabil. Eng., vol. 23, no. 1, pp. 51-63, 2015.

[20] S. L. Norman et al., "Planar hand motion guidance using fingertip skin-stretch feedback," IEEE Trans. Haptics, vol. 7, no. 2, pp. 121130, 2014.

[21] K. Kim and J. E. Colgate, "Haptic feedback enhances grip force control of semg-controlled prosthetic hands in targeted reinnervation amputees," IEEE Trans. Neural Syst. and Rehabil. Eng., vol. 20, no. 6, pp. 798-805, 2012.

[22] E. Pezent et al., "Design and Characterization of the OpenWrist: A Robotic Wrist Exoskeleton for Coordinated Hand-wrist Rehabilitation," in IEEE Intl. Conf. Rehabil. Robot. (ICORR), 2017.

[23] J. A. French et al., "System characterization of MAHI Exo-II: A robotic exoskeleton for upper extremity rehabilitation," in ASME Dynamic Systems and Control Conf., 2014.

[24] S. Casini et al., "Design and realization of the cuff-clenching upperlimb force feedback wearable device for distributed mechano-tactile stimulation of normal and tangential skin forces," in IEEE Intl. Conf. Intell. Robots and Syst. (IROS), 2015, pp. 1186-1193.

[25] S. B. Godfrey et al., "Influence of force feedback on grasp force modulation in prosthetic applications: A preliminary study," in IEEE Intl. Conf. Eng. in Medicine and Biology Society (EMBC), 2016, pp. 5439-5442.

[26] S. Fani et al., "Integrating wearable haptics and teleimpedance methods for augmented human-robot interaction with synergy-inspired robotic systems," Robotic \& Automation Magazine, Accepted, 2017.

[27] K. J. Verhoeven, K. L. Simonsen, and L. M. McIntyre, "Implementing false discovery rate control: Increasing your power," Oikos, vol. 108, no. 3, pp. 643-647, 2005.

[28] J. D. Brown et al., "Co-location of force and action improves identification of force-displacement features," in IEEE Haptics Symposium, March 2012, pp. 187-193. 